\title{
The pre-outburst flare of the A $0535+26$ August/September 2005 outburst ${ }^{\star}$
}

\author{
I. Caballero ${ }^{1}$, A. Santangelo ${ }^{1}$, P. Kretschmar ${ }^{2}$, R. Staubert ${ }^{1}$, K. Postnov ${ }^{1,3}$, D. Klochkov ${ }^{1}$, A. Camero-Arranz ${ }^{4}$, \\ M. H. Finger ${ }^{5}$, I. Kreykenbohm ${ }^{1,6}$, K. Pottschmidt ${ }^{7,8}$, R. E. Rothschild ${ }^{9}$, S. Suchy ${ }^{9}$, J .Wilms ${ }^{10}$, and C. A. Wilson ${ }^{5}$ \\ 1 Institut für Astronomie und Astrophysik, Sand 1, 72076 Tübingen, Germany \\ e-mail: isabel@astro.uni-tuebingen.de \\ 2 ISOC, European Space Astronomy Centre (ESAC), PO Box 78, 28691 Villanueva de la Cañada, Madrid, Spain \\ 3 Sternberg Astronomical Institute, 119999 Moscow, Russia \\ ${ }^{4}$ GACE, Instituto de Ciencias de los Materiales, Universidad de Valencia, PO Box 20085, 46071 Valencia, Spain \\ 5 National Space Science and Technology Center, 320 Sparkman Drive NW, Huntsville, AL 35805, USA \\ 6 ISDC, 16 Ch. d'Écogia, 1290 Versoix, Switzerland \\ 7 CRESST, University of Maryland Baltimore County, 1000 Hilltop Circle, Baltimore, MD 21250, USA \\ 8 NASA Goddard Space Flight Center, Astrophysics Science Division, Code 661, Greenbelt, MD 20771, USA \\ 9 CASS, University of California at San Diego, La Jolla, CA 92093-0424, USA \\ 10 Dr. Remeis-Sternwarte, Astronomisches Institut der Universität Erlangen-Nürnberg, Sternwartstr. 7, 96049 Bamberg, Germany \\ Received 21 December 2007 / Accepted 8 January 2008
}

\begin{abstract}
Aims. We study the spectral and temporal behavior of the High Mass X-ray Binary A 0535+26 during a "pre-outburst flare" which took place $\sim 5 \mathrm{~d}$ before the peak of a normal (type I) outburst in August/September 2005. We compare the studied behavior with that observed during the outburst.

Methods. We analyse RXTE observations that monitored A 0535+26 during the outburst. We complete spectral and timing analyses of the data. We study the evolution of the pulse period, present energy-dependent pulse profiles both at the initial pre-outburst flare and close to outburst maximum, and measure how the cyclotron resonance-scattering feature (hereafter CRSF) evolves.

Results. We present three main results: a constant period $P=103.3960(5) \mathrm{s}$ is measured until periastron passage, followed by a spin-up with a decreasing period derivative of $\dot{P}=(-1.69 \pm 0.04) \times 10^{-8} \mathrm{~s} \mathrm{~s}^{-1}$ at MJD 53 618, and $P$ remains constant again at the end of the main outburst. The spin-up provides evidence for the existence of an accretion disk during the normal outburst. We measure a CRSF energy of $E_{\mathrm{cyc}} \sim 50 \mathrm{keV}$ during the pre-outburst flare, and $E_{\mathrm{cyc}} \sim 46 \mathrm{keV}$ during the main outburst. The pulse shape, which varies significantly during both pre-outburst flare and main outburst, evolves strongly with photon energy.
\end{abstract}

Key words. X-rays: binaries - stars: magnetic fields - stars: individual: A 0535+26

\section{Introduction}

The Be/X-ray binary A $0535+26^{1}$ is a transient source, characterized by quiescent states with $\mathrm{X}$-ray luminosity $L_{\mathrm{X}} \lesssim$ $10^{36} \mathrm{erg} \mathrm{s}^{-1}$, interrupted by normal (type I) outbursts, associated in general with periaston passage, when a luminosity $L_{\mathrm{X}} \sim 10^{36-37} \mathrm{erg} \mathrm{s}^{-1}$ is reached, and giant (type II) outbursts for which $L_{\mathrm{X}}>10^{37} \mathrm{erg} \mathrm{s}^{-1}$. A $0535+26$ was discovered by Rosenberg et al. (1975) during a giant outburst of luminosity level of $L_{(3-7 \mathrm{keV})} \sim 1.2 \times 10^{37} \mathrm{erg} \mathrm{s}^{-1}$. Since then, five giant outbursts have been detected in October 1980 (Nagase et al. 1982), June 1983 (Sembay et al. 1990), March/April 1989 (Makino et al. 1989), February 1994 (Finger et al. 1994), and May/ June 2005 (Tueller et al. 2005). Following the giant outburst in May/June 2005, two normal outbursts occurred in August/ September 2005 (Finger 2005b) and December 2005 (Finger 2005a).

The source A $0535+26$ is in a binary orbit, with an O9.7IIIe optical companion HDE 245770 (Giangrande et al. 1980), of orbital period $P_{\text {orb }}=110.3 \pm 0.3$ days and

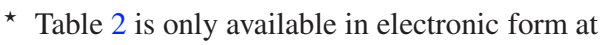
http: //www . aanda. org

${ }^{1}$ Referred to as $1 \mathrm{~A} 0535+262$ in SIMBAD.
}

eccentricity $e=0.47 \pm 0.02$ (Finger et al. 1994). The estimated distance of the system is $2 \mathrm{kpc}$ (Steele et al. 1998). The measured pulse period of the neutron star at MJD 53614.5137 is $P=103.39315(5)$ s (Caballero et al. 2007). During quiescence a spin-down trend of the neutron star has been reported (Finger et al. 1996; Hill et al. 2007), and during giant outbursts the neutron star shows a strong spin-up. In the June 1983 giant outburst, a spin-up of $\dot{v} \sim 0.6 \times 10^{-11} \mathrm{~Hz} \mathrm{~s}^{-1}$ was measured (Sembay et al. 1990). In the February 1994 giant outburst the spin-up reached $\dot{v} \sim 1.2 \times 10^{-11} \mathrm{~Hz} \mathrm{~s}^{-1}$ and quasi periodic oscillations were present, confirming the presence of an accretion disk (Finger et al. 1996).

The X-ray spectrum of A $0535+26$ is typically described by a phenomenological model consisting of a power law with an exponential cutoff. Two absorption like features, interpreted as cyclotron resonance scattering features CRSFs (fundamental and first harmonic), have been observed in the spectrum at $\sim 45 \mathrm{keV}$ and $\sim 100 \mathrm{keV}$ (Kendziorra et al. 1994; Grove et al. 1995). Recent observations during the August/September 2005 normal outburst with INTEGRAL and RXTE (Kretschmar et al. 2005; Wilson \& Finger 2005; Caballero et al. 2007) and Suzaku (Terada et al. 2006) have confirmed this result. The centroid energy of the CRSF was measured during the main outburst at 


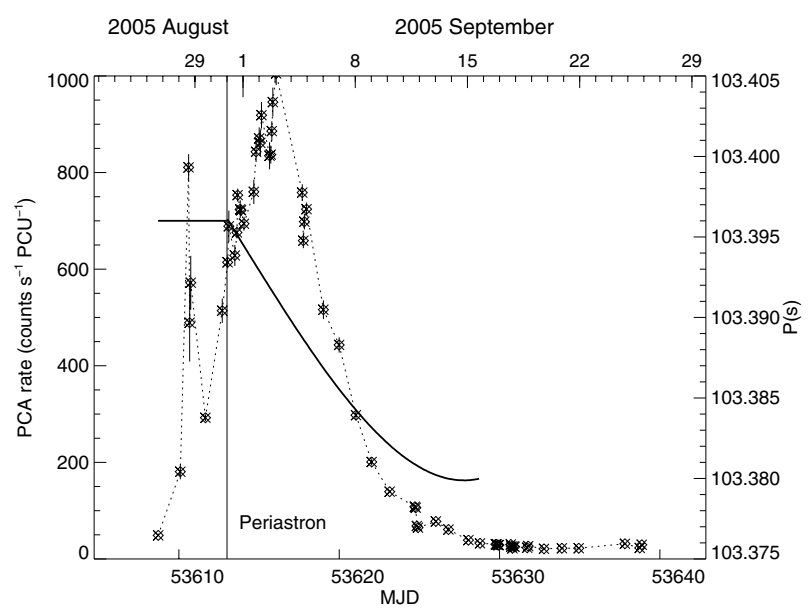

Fig. 1. Left axis, dotted line: $\sim 3-30 \mathrm{keV}$ PCA mean count rate for each available observation during the August/September 2005 outburst. Each diamond indicates one RXTE observation. The mean was obtained averaging each individual light curve. The errors shown are the sigma of the distribution for each observation. Right axis, solid line: pulse period evolution during the outburst. The vertical line indicates the time of periastron.

different luminosity levels and it was not found to vary with Xray luminosity.

In this Letter we focus on the August/September 2005 normal outburst. At the onset of this outburst, a sharp, pre-outburst flare is observed superimposed on the gradual increase towards the peak flux in the main outburst. This pre-outburst flare develops on a time scale of 1 day, short compared to the threeweek duration of the main outburst. As can be seen from the light curve measured by Swift/BAT, the flare is one of several such flares superimposed on the rising edge of the main outburst (see Postnov et al. 2008). The pre-outburst flare studied here reaches a PCA flux of 810 counts $\mathrm{s}^{-1} \mathrm{PCU}^{-1}$, and the maximum flux during the main outburst is 1005 counts s${ }^{-1} \mathrm{PCU}^{-1}$. The spectral and timing behaviours of the source during the preoutburst flare appear to be different from the ones observed during the main outburst.

\section{Instruments and observations}

RXTE (Bradt et al. 1993) observed A 0535+26 between 2005 August 28 and 2005 September 24 in a target of opportunity observation completed as part of a campaign studying accreting pulsars. We present observations from the Proportional Counter Array PCA (2-60 keV, Jahoda et al. 1996) and from the High Energy X-ray Timing Experiment HEXTE $(20-200 \mathrm{keV}$, Rothschild et al. 1998). A total of 44 pointed observations were completed to monitor the outburst with a total exposure time of $\sim 140$ ks. Figure 1 shows the PCA light curve during the outburst in the $\sim 3-30 \mathrm{keV}$ range, obtained by averaging the individual light curves of all the observations. The analysis of RXTE data was performed with FTOOLS 6.3.1 and the spectral analysis with the X-Ray Spectral Fitting Package XSPEC v11 (Arnaud 1996).

\section{Timing analysis}

\subsection{Evolution of pulse period during the outburst}

We measured the pulse period of the pulsar with high accuracy during the outburst. For each available observation, we extracted
Table 1. Formal function decribing the pulse period during the outburst. The reference for the third order polinomial fit is MJD 53618. From MJD 53629 the period is essentially constant.

\begin{tabular}{ccc}
\hline \hline & MJD & MJD \\
& $53608.70-53613.02$ & $53613.11-53629$ \\
\hline$P(\mathrm{~s})$ & $103.3960(5)$ & $103.3883(5)$ \\
$\dot{P}\left(\mathrm{~s} \mathrm{~s}^{-1}\right)$ & - & $(-1.69 \pm 0.04) \times 10^{-8}$ \\
$\ddot{P}\left(\mathrm{~s} \mathrm{~s}^{-2}\right)$ & - & $(9 \pm 3) \times 10^{-15}$ \\
$\mathrm{~d}^{3} P / \mathrm{d} t^{3}\left(\mathrm{~s} \mathrm{~s}^{-3}\right)$ & - & $(2.5 \pm 0.9) \times 10^{-20}$ \\
\hline
\end{tabular}

barycentric-corrected PCA and HEXTE light curves and corrected the photon arrival times for the orbital motion. We used the orbital ephemeris from Finger et al. (1994) with the periastron epoch updated to MJD $53613.0 \pm 1.3$ (Finger et al. 2006, 2007). We then produced pulse profiles using a constant, trial period obtained using INTEGRAL observations of the same outburst (Caballero et al. 2007). For both PCA and HEXTE we determined a reference time in the pulse profiles, and performed a phase-connection analysis as previously completed in Caballero et al. (2007, see also Ferrigno et al. 2007). As a phase reference we selected the midpoint of a sharp edge present in the pulse profiles, an apparently stable feature. The pulse period is approximately constant during the initial pre-outburst flare, and a significant spin-up is measured after periastron. The period evolution after periastron can be described by a third-order polynomial. The results of the fit are provided in Table 1 . The period at any time during the outburst is well-described using those functions, and $\pm 5 \mathrm{~ms}$ is a conservative estimate of the uncertainty. The function describing the period evolution is plotted in Fig. 1. It is consistent with the period measured using INTEGRAL data within uncertainties (Caballero et al. 2007).

\subsection{Pulse profile evolution during the outburst}

Applying the measured period and its derivatives to fold light curves, we studied changes in the pulse profiles between the pre-outburst flare and the main outburst. Strong changes in the pulse-profile shape, with photon energy, are observed for both the main outburst and pre-outburst flare but in quite different ways. Figure 2 shows pulse profiles from observations (a), during the pre-outburst flare, and (b), close to the maximum of the main outburst. Observation (b) was chosen because of its high data quality and the pulse profiles are representative of data acquired during the outburst peak. The low-energy pulse profiles in both cases show a complex pattern, but different structures and evolution. At higher energies both pulse profiles show a simpler two peaked shape. During the main outburst, one of the peaks is strongly reduced at the cyclotron energy. During the pre-outburst flare there is a smooth evolution towards higher energies without a change crossing the cyclotron energy. Energy-dependent pulse profiles for all the observations during the outburst are shown in Camero-Arranz (2007) and a detailed study will be presented in a forthcoming paper (Camero-Arranz et al. 2007).

\section{Spectral analysis}

To model the phase-averaged, X-ray continuum, we have used a phenomenological model, a power law times an exponential cutoff (XSPEC model cutoffpl), which is the simplest capable

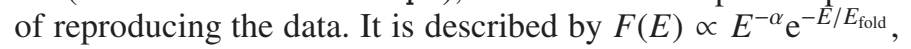
where $\alpha$ is the photon index and $E_{\text {fold }}$ the folding energy. One, and in some observations two, absorption features were included in the model to enable an accurate description of the data. We 


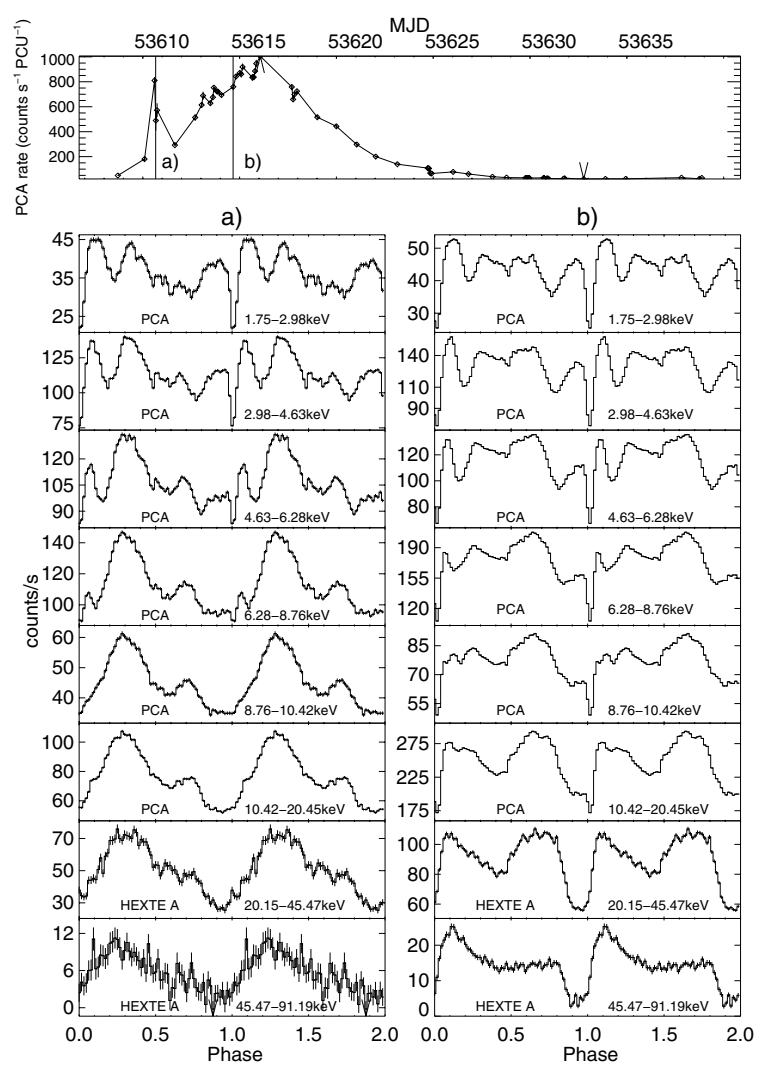

Fig. 2. $P C A$ and HEXTE background substracted pulse profiles from one observation during the pre-periastron flare a), with $L_{(5-50 \mathrm{keV})} \sim$ $0.45 \times 10^{37} \mathrm{erg} \mathrm{s}^{-1}$, and from one observation near the maximum of the outburst b), with $L_{(5-50 \mathrm{keV})} \sim 0.75 \times 10^{37} \mathrm{erg} \mathrm{s}^{-1}$. Two pulse cycles are shown for clarity. In the upper panel the PCA light curve is shown. The vertical lines indicate the corresponding observations. The exposure times are $2.2 \mathrm{ks}$ in a) and $12.35 \mathrm{ks}$ in $\mathbf{b}$ ).

modeled them with a Gaussian optical depth profile, which modifies the continuum in the following way: $F^{\prime}(E)=F(E) \mathrm{e}^{-\tau(E)}$, where $\tau(E)=\tau \mathrm{e}^{-\left(E-E_{\text {cyc }}\right)^{2} /\left(2 \sigma^{2}\right)}$. A Gaussian emission line was added to the model to account for the $\mathrm{Fe} \mathrm{K} \alpha$ fluorescence line, with energy fixed at $6.4 \mathrm{keV}$ and width to $0.5 \mathrm{keV}$. Due to a feature in the residuals around $\sim 4.7 \mathrm{keV}$ (instrumental Xenon L edge, see Rothschild et al. 2006), we excluded data for energies below $5 \mathrm{keV}$ in our analysis.

We performed a spectral analysis of all observations available and studied the evolution of the cyclotron-line energy and continuum parameters during the outburst. We measured a constant value for the centroid energy of the fundamental CRSF during the main outburst, as reported by Caballero et al. (2007). During the pre-outburst flare, the cyclotron-centroid energy is measured at a higher value, reaching $E_{\mathrm{cyc}}=52.0_{-1.4}^{+1.6} \mathrm{keV}$, compared to $E_{\text {cyc }}=46.1_{-0.5}^{+0.5} \mathrm{keV}$ during the main outburst (at $90 \%$ confidence). To study the significance of the change, we have produced $\chi^{2}$ contour plots for the observations during the flare and during the main outburst. As an example, in Fig. 3 contour plots are shown for the observation close to the maximum of the main outburst labeled (b) in Fig. 2, and for the sum of the three available observations during the pre-outburst flare. In observation (b) we included a harmonic, cyclotron line with the energy fixed at $E_{\mathrm{cyc}}=102.5 \mathrm{keV}$ (it is measured at $E_{\mathrm{cyc}}=102.5_{-3.3}^{+4.5} \mathrm{keV}$ at $90 \%$ confidence). We conclude from the contour plots that the change in energy is statistically significant.

Table 2 contains the best-fit values for the three observations during the pre-outburst flare and for the sum of those
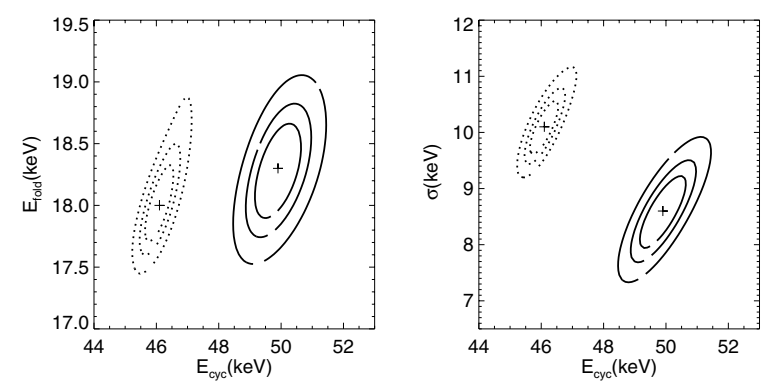

Fig. 3. $E_{\text {fold }}$ vs. $E_{\text {cyc }}(l e f t)$ and $\sigma$ vs. $E_{\text {cyc }}$ (right) contour plots for one observation near the maximum (dotted lines, observation (b) in Fig. 2) and for the sum of the three available observations during the pre-outburst flare (solid lines). The contours indicate $\chi_{\min }^{2}+$ 2.30(68\%), 4.61(90\%), 9.21(99\%) levels.

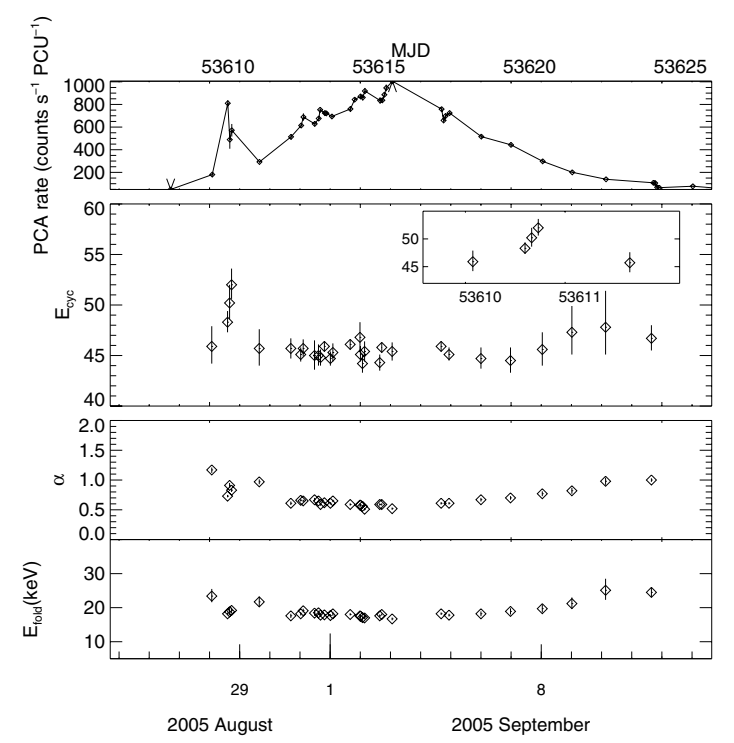

Fig. 4. First panel: PCA light curve. Second panel: fundamental CRSF energy during the ourbusrt. Inset shows a zoom on the pre-outburst flare. Third and forth panels: photon index and folding energy evolution. Errors are $90 \%$ confidence for one parameter of interest $\left(\chi_{\min }^{2}+2.7\right)$.

observations, as well as the best-fit values for the main outburst (observation (b) in Fig. 2).

Figure 4 shows the evolution of the CRSF energy during the outburst, as well as the evolution of the continuum parameters photon index $\alpha$ and folding energy $E_{\text {fold }}$ for the observations in which the cyclotron line was detected. The continuum parameters are quite variable at the beginning of the outburst. The spectrum at the beginning of the flare appears to be softer, with photon index $\alpha \sim 1.2$, and then becomes harder in the flare. The rising of the main outburst presents a harder spectrum, with $\alpha \sim 0.6$. The decay of the outburst shows a smooth softening of the spectrum.

\section{Summary and discussion}

In this paper, we present evidence for significant changes in the spectral and timing parameters of the Be/X-ray binary A 0535+26, during its normal outburst in August/ September 2005. Our three main results are the following:

1. The pulse period of the neutron star appears to be constant during the pre-outburst flare, $P=103.3960(5) \mathrm{s}$, and a spinup starts at periastron, $\dot{P}=(-1.69 \pm 0.04) \times 10^{-8} \mathrm{~s} \mathrm{~s}^{-1}$ measured at MJD 53618 . The pulse period falls exponentially at the end of the outburst. 
2. The energy-dependent, pulse profiles during the pre-outburst flare are significantly different from those measured for the main outburst.

3. During the pre-outburst flare, the fundamental cyclotron-line energy centroid reaches $E_{\text {cyc }}=52.0_{-1.4}^{+1.6} \mathrm{keV}$, significantly higher than for the main outburst, $E_{\mathrm{cyc}}=46.1_{-0.5}^{+0.5} \mathrm{keV}$.

This is the first observation of a normal outburst that measures a spin-up rate for A $0535+26$, providing evidence that an accretion disk is being detected during a type I outburst. The measured spin-up $\dot{P}=(-1.69 \pm 0.04) \times 10^{-8} \mathrm{~s} \mathrm{~s}^{-1}$, or $\dot{v}=(1.58 \pm 0.04) \times$ $10^{-12} \mathrm{~Hz} \mathrm{~s}^{-1}$, is smaller than the spin-up measured during giant outbursts in the past, e.g., $\dot{v} \sim 0.6 \times 10^{-11} \mathrm{~Hz} \mathrm{~s}^{-1}$ in June 1983 (Sembay et al. 1990), or $\dot{v} \sim 1.2 \times 10^{-11} \mathrm{~Hz} \mathrm{~s}^{-1}$ in February 1994 (Finger et al. 1996).

The pulse profiles measured during the main outburst are consistent with those during a giant outburst in March/April 1989 albeit at a different luminosity level, $L_{(23-53 \mathrm{keV})} \sim 1.26 \times 10^{37} \mathrm{erg} \mathrm{s}^{-1}$ (Kendziorra et al. 1994). A comparison between these pulse profiles and those obtained for the pre-outburst flare in this paper suggest that a different mode of accretion takes place during the flare (see Postnov et al. 2008).

The energy of the CRSF during the main outburst is consistent with the energy measured with INTEGRAL close to the maximum of the main outburst, $E_{\text {cyc }}=45.9 \pm 0.3 \mathrm{keV}$ (Caballero et al. 2007) and with the energy measured with Suzaku at the end of the main outburst, $E_{\text {cyc }}=46.3_{-1.3}^{+1.5} \mathrm{keV}$ (using the same Gaussian model for the line, Terada et al. 2006). From the observed cyclotron energy, the estimated, magnetic field at the site of the X-ray emission during the main outburst, assuming a redshift of $z=0.3$, is $B \sim 5.2 \times 10^{12} \mathrm{G}$, and during the pre-outburst flare $B \sim 5.8 \times 10^{12} \mathrm{G}$. During the main part of the outburst the cyclotron-line energy remains constant within the errors, in spite of the changes in the luminosity. This suggests that the structure of the accretion column is different from that observed in both V $0332+53$ (Mowlavi et al. 2006) and 4U 0115+63 (Tsygankov et al. 2006). The source is likely to be in the sub-Eddington regime, as for Her X-1 (Staubert et al. 2007) for which it is believed no shock has formed in the accretion column.

Pre-outburst flares have been observed in other accreting pulsars, such as 2S 1845-024 (Finger et al. 1999) and EXO 2030+375 (Camero-Arranz et al. 2005).

Hayasaki \& Okazaki (2006) have modeled accretion disks around neutron stars in Be/X-ray binaries. Their SPH simulations reproduce a series of normal outbursts from the accretion disk formed about the neutron star at periastron. In some cases, single flares are found preceding the outburst maximum. We note first of all that the timescale of the flare in their simulations appears to be too long ( $10 \%$ of the orbital period) compared to the flare duration observed. The inspection of the Swift-BAT light-curve of A0535+26 during the outburst studied here shows that RXTE actually observed only one of a collection of short flares in the rising part of the outburst (Postnov et al. 2008). Such flares are not reproduced by the above simulations. This model does not take into account the disk-magnetospheric interaction.
A more plausible interpretation is that the pre-outburst flares could be caused by magnetospheric instabilities between the accretion disk and the neutron-star magnetosphere at the onset of accretion. The instability causes the plasma that has accumulated in the disk-magnetosphere, boundary-layer to rapidly fall onto the neutron star surface close to the magnetic poles, but along different field lines than (quasi)stationary accretion (see Postnov et al. 2008, for details).

Acknowledgements. We thank ISSI (Bern), for their hospitality during the team meetings held there. I.C. thanks ESAC (ESA) in Madrid for their hospitality. This research is supported by the Bundesministerium für Wirtschaft und Technologie through the German Space Agency (DLR) under contract No. 50 OR 0302.

\section{References}

Arnaud, K. A. 1996, in Astronomical Data Analysis Software and Systems V, ed. G. H. Jacoby, \& J. Barnes, ASP Conf. Ser., 101, 17

Bradt, H. V., Rothschild, R. E., \& Swank, J. H. 1993, A\&AS, 97, 355

Caballero, I., Kretschmar, P., Santangelo, A., et al. 2007, A\&A, 465, L21 Camero-Arranz, A. 2007, Ph.D. Thesis, Universidad de Valencia

Camero-Arranz, A., Wilson, C. A., Connell, P., et al. 2005, A\&A, 441, 261 Camero-Arranz, A., et al. 2007, in preparation

Ferrigno, C., Segreto, A., Santangelo, A., et al. 2007, A\&A, 462, 995

Finger, M. H. 2005a, ATel, 676

Finger, M. H. 2005b, ATel, 595

Finger, M. H., Bildsten, L., Chakrabarty, D., et al. 1999, ApJ, 517, 449

Finger, M. H., Cominsky, L. R., Wilson, R. B., Harmon, B. A., \& Fishman, G. J. 1994, in The Evolution of X-ray Binaries, ed. S. Holt \& C. S. Day, AIP Conf. Proc. 308, 459

Finger, M. H., Wilson, R. B., \& Harmon, B. A. 1996, ApJ, 459, 288

Finger, M. H., Camero-Arranz, A., Kretschmar, P., Wilson, C., \& Patel, S. 2006, BAAS, 38, 359

Finger, M. H., et al. 2007, in preparation

Giangrande, A., Giovannelli, F., Bartolini, C., Guarnieri, A., \& Piccioni, A. 1980, A\&AS, 40, 289

Grove, J. E., Strickman, M. S., Johnson, W. N., et al. 1995, ApJ, 438, L25

Hayasaki, K., \& Okazaki, A. T. 2006, MNRAS, 372, 1140

Hill, A. B., Bird, A. J., Dean, A. J., et al. 2007, MNRAS, 381, 1275

Jahoda, K., Swank, J. H., Giles, A. B., et al. 1996, in EUV, X-Ray, and Gamma-

Ray Instrumentation for Astronomy VII, ed. O. H. Siegmund, \& M. A. Gummin, Proc. SPIE, 2808, 59

Kendziorra, E., Kretschmar, P., Pan, H. C., et al. 1994, A\&A, 291, L31

Kretschmar, P., Kreykenbohm, I., Pottschmidt, K., et al. 2005, ATel, 601

Makino, F., Cook, W., Grunsfeld, J., et al. 1989, IAU Circ., 4769

Mowlavi, N., Kreykenbohm, I., Shaw, S. E., et al. 2006, A\&A, 451, 187

Nagase, F., Hayakawa, S., Kunieda, H., et al. 1982, ApJ, 263, 814

Postnov, K., Staubert, R., Santangelo, A., et al. 2008, A\&A, 480, L21

Rosenberg, F. D., Eyles, C. J., Skinner, G. K., \& Willmore, A. P. 1975, Nature, 256, 628

Rothschild, R. E., Blanco, P. R., Gruber, D. E., et al. 1998, ApJ, 496, 538

Rothschild, R. E., Wilms, J., Tomsick, J., et al. 2006, ApJ, 641, 801

Sembay, S., Schwartz, R. A., Orwig, L. E., Dennis, B. R., \& Davies, S. R. 1990, ApJ, 351, 675

Staubert, R., Shakura, N. I., Postnov, K., et al. 2007, A\&A, 465, L25

Steele, I. A., Negueruela, I., Coe, M. J., \& Roche, P. 1998, MNRAS, 297, L5

Terada, Y., Mihara, T., Nakajima, M., et al. 2006, ApJ, 648, L139

Tsygankov, S. S., Lutovinov, A. A., Churazov, E. M., \& Sunyaev, R. A. 2006, MNRAS, 371, 19

Tueller, J., Ajello, M., Barthelmy, S., et al. 2005, ATel, 504

Wilson, C. A., \& Finger, M. H. 2005, ATel, 605 
I. Caballero et al.: Pre-outburst flare of A 0535+26, Online Material p 1

Table 2. Best-fit values for the available observations during the pre-outburst flare, where the CRSF energy is measured at a higher position, for the sum of the observations during the flare, and for one observation close to the maximum of the main outburst. $L$ is the luminosity in units of $10^{37} \mathrm{erg} \mathrm{s}^{-1}$ in the $5-50 \mathrm{keV}$ range. Errors are $90 \%$ confidence for one parameter of interest $\left(\chi_{\min }^{2}+2.7\right)$.

\begin{tabular}{|c|c|c|c|c|c|c|c|c|c|}
\hline Obs. ID & $E_{\text {cyc }}(\mathrm{keV})$ & $\sigma(\mathrm{keV})$ & $\tau$ & $E_{\text {fold }}(\mathrm{keV})$ & $\alpha$ & MJD start & Exp. (ks) & $L$ & $\chi_{\text {red }}^{2} /$ d.o.f. \\
\hline 91086-01-03-01 & $48.3_{-1.0}^{+1.1}$ & $6.4_{-1.0}^{+1.0}$ & $0.27_{-0.03}^{+0.03}$ & $18.2_{-0.4}^{+0.4}$ & $0.73_{-0.02}^{+0.02}$ & 53610.599 & 1.152 & 0.85 & $1.19 / 170$ \\
\hline 91086-01-03-02 & $50.2_{-1.6}^{+1.8}$ & $8.7_{-1.3}^{+1.4}$ & $0.57_{-0.08}^{+0.08}$ & $18.8_{-1.0}^{+1.0}$ & $0.91_{-0.04}^{+0.04}$ & 53610.665 & 2.208 & 0.45 & $0.88 / 170$ \\
\hline 91086-01-03-03 & $52.0_{-1.4}^{+1.6}$ & $10.8_{-1.1}^{+1.3}$ & $0.53_{-0.06}^{+0.06}$ & $19.2_{-0.7}^{+0.8}$ & $0.83_{-0.03}^{+0.03}$ & 53610.729 & 2.560 & 0.57 & $0.90 / 170$ \\
\hline sum & $49.9_{-0.8}^{+0.8}$ & $8.6_{-0.7}^{+0.7}$ & $0.40_{-0.03}^{+0.03}$ & $18.3_{-0.4}^{+0.4}$ & $0.77_{-0.02}^{+0.02}$ & 53610.599 & 5.920 & 0.66 & $1.18 / 220$ \\
\hline $91085-01-01-03$ & $\begin{array}{c}46.1_{-0.5}^{+0.5} \\
102.5\end{array}$ & $\begin{array}{c}10.1_{-0.5}^{+0.5} \\
8.4_{-1.9}^{+2.2}\end{array}$ & $\begin{array}{c}0.47_{-0.02}^{+0.03} \\
1.0_{-0.2}^{+0.4}(1 \mathrm{st} \text { harmonic })\end{array}$ & $18.0_{-0.3}^{+0.4}$ & $0.59_{-0.02}^{+0.02}$ & 53614.7 & 12.352 & 0.75 & $1.19 / 214$ \\
\hline
\end{tabular}

